\title{
Some Remarks on Second Order Linear Difference Equations*
}

\author{
B.G. ZHANG $^{\mathrm{a}, \dagger}$ and CHUAN JUN TIAN ${ }^{\mathrm{b}}$ \\ ${ }^{\text {a }}$ Department of Applied Mathematics, Ocean University of Qingdao, Qingdao 266003, P.R. China; \\ ${ }^{\mathrm{b}}$ Department of Mathematics, Jinzhou Teacher's College, Jinzhou, Hubei 434100, P.R. China
}

(Received 20 August 1997)

\begin{abstract}
We obtain some further results for comparison theorems and oscillation criteria of second order linear difference equations.
\end{abstract}

Keywords: Oscillations, Comparison theorems, Difference equations

AMS Subject Classifications: 39A10

\section{INTRODUCTION}

Oscillation and comparison theorems for the linear difference equation

$$
c_{n} x_{n+1}+c_{n-1} x_{n-1}=b_{n} x_{n}, \quad n=1,2, \ldots,
$$

has been investigated intensively [1-5].

Equation (1.1) is equivalent to the self adjoint equation

$$
\Delta\left(c_{n-1} \Delta x_{n-1}\right)+a_{n} x_{n}=0, \quad n=1,2, \ldots,
$$

where $a_{n}=c_{n}+c_{n-1}-b_{n}$.

A nontrivial solution $\left\{x_{n}\right\}$ of Eq. (1.1) is said to be oscillatory, if the terms $x_{n}$ of the solution are neither eventually all positive nor eventually all negative. Otherwise, the solution is called nonoscillatory. It is well known that if one nontrivial solution of (1.1) is oscillatory, then all solutions are oscillatory, and so we can say that (1.1) is oscillatory.

In Section 2, we want to show some further results on the comparison theorem and oscillation criteria for (1.1), which improve some known results. In Section 3, we consider the forced oscillation.

\section{COMPARISON THEOREMS AND OSCILLATION}

We assume that $a_{n}>0$ and $b_{n}>0$ for all large $n$. Let $\left\{x_{n}\right\}$ be an eventually positive solution of (1.1),

* The research was supported by NNSF of China.

${ }^{\dagger}$ Corresponding author. 
say $x_{n}>0$ for $n \geq N$. Taking Riccati type transformation

$$
s_{n}=\left(b_{n+1} x_{n+1}\right) /\left(c_{n} x_{n}\right), \quad n \geq N,
$$

(1.1) becomes

$$
q_{n} s_{n}+1 / s_{n-1}=1, \quad \text { for } n \geq N+1,
$$

where $q_{n}=c_{n}^{2} /\left(b_{n} b_{n+1}\right)$.

It is known [1] that (1.1) is nonoscillatory if and only if (2.2) has an eventually positive solution.

We consider (1.1) and (2.2) together with

$$
C_{n} y_{n+1}+C_{n-1} y_{n-1}=B_{n} y_{n}, \quad n=1,2, \ldots,
$$

and

$$
Q_{n} S_{n}+1 / S_{n-1}=1,
$$

where $Q_{n}=C_{n}^{2} /\left(B_{n} B_{n+1}\right)$.

THEOREM 2.1 Suppose that $Q_{n} Q_{n+1} \geq q_{n} q_{n+1}$ and $Q_{n}+Q_{n+1} \geq q_{n}+q_{n+1}$ for all large $n$. If (2.3) is nonoscillatory, so is Eq. (1.1).

Proof To prove that (1.1) has a positive solution, it is sufficient to prove that (2.2) has a positive solution $\left\{s_{n}\right\}$ for $n \geq N$. Since $q_{n}+q_{n+1} \leq$ $Q_{n}+Q_{n+1}$ for all large $n$, then there exists a positive integer $n_{1}>N$ such that $Q_{n_{1}+1} \geq q_{n_{1}+1}$. From (2.4), $S_{n}>1$ for $n \geq n_{1}$. Choose $s_{n_{1}} \geq S_{n_{1}}>1$ and define $s_{n_{1}+1}$ by (2.2). In view of (2.2) and (2.4), we have

$$
\begin{aligned}
q_{n_{1}+1} s_{n_{1}+1}= & 1-1 / s_{n_{1}} \\
= & Q_{n_{1}+1} S_{n_{1}+1}+1 / S_{n_{1}} \\
& -1 / s_{n_{1}} \geq Q_{n_{1}+1} S_{n_{1}+1}
\end{aligned}
$$

Hence

$$
S_{n_{1}+1} \geq \frac{Q_{n_{1}+1}}{q_{n_{1}+1}} S_{n_{1}+1}>0
$$

and $s_{n_{1}} s_{n_{1}+1} \geq S_{n_{1}} S_{n_{1}+1}$. By induction, we can prove that (2.2) has a positive solution $\left\{s_{n}\right\}$, $n \geq n_{1}$, which implies that (1.1) has a nonoscillatory solution. The proof is complete.

Remark 2.1 Theorem 2.1 improves Theorem 6.8.4 in [1].
We write (1.1) in the form

$$
x_{n+1}-\frac{b_{n}}{c_{n}} x_{n}+\frac{c_{n-1}}{c_{n}} x_{n-1}=0
$$

and let $y_{n}=\left[\prod_{i=N}^{n-1}\left(c_{i} / b_{i}\right)\right] x_{n}$. Then (1.1) becomes

$$
y_{n+1}-y_{n}+q_{n} y_{n-1}=0 \text {. }
$$

The oscillation of (1.1) and (2.5) is equivalent. By known results [1, Theorems 6.20 .3 and 6.20.4] or [2], if

$$
\liminf _{n \rightarrow \infty} q_{n}>\frac{1}{4}
$$

then (1.1) is oscillatory and if

$$
\limsup _{n \rightarrow \infty} q_{n}<\frac{1}{4}
$$

then (1.1) is nonoscillatory. In particular, the equation

$$
y_{n+1}-y_{n}+\frac{1}{4} y_{n-1}=0
$$

is nonoscillatory.

Combining the above results and Theorem 2.1, we obtain the following corollaries.

COROLlaRY 2.1 If $q_{n}+q_{n+1} \leq 1 / 2$ for all large n. Then (1.1) is nonoscillatory.

In fact, let $Q_{n} \equiv 1 / 4$, Corollary 2.1 follows from Theorem 2.1 .

Remark 2.2 Corollary 2.1 improves Theorem 6.5.5 in [1].

COROLlary 2.2 If $q_{n} q_{n+1} \geq 1 / 16+\epsilon_{0}$, for some $\epsilon_{0}>0$ and all large $n$, then (1.1) is oscillatory.

Proof Let $\epsilon_{1}$ be a positive number such that $1 /\left(4-\epsilon_{1}\right) \leq \sqrt{1 / 16+\epsilon_{0}}$ and $Q_{n}=1 /\left(4-\epsilon_{1}\right)$ for all large $n$. Then

$$
q_{n} q_{n+1} \geq \frac{1}{16}+\epsilon_{0} \geq \frac{1}{\left(4-\epsilon_{1}\right)^{2}}=Q_{n} Q_{n+1}
$$

and

$$
q_{n}+q_{n+1} \geq 2 \sqrt{q_{n} q_{n+1}} \geq \frac{2}{4-\epsilon_{1}}=Q_{n}+Q_{n+1}
$$


for all large $n$. Since $Q_{n} \equiv 1 /\left(4-\epsilon_{1}\right)$ implies that (2.3) is oscillatory. By Theorem 2.1, (1.1) is oscillatory also.

Remark 2.3 Corollary 2.2 improves Theorem 6.5.3 in [1].

Example 2.1 Consider the difference equation

$$
c_{n} x_{n+1}+c_{n-1} x_{n-1}=x_{n},
$$

where

$$
c_{n-1}= \begin{cases}\sqrt{14.1 / 15}, & n: \text { even }, \\ \sqrt{1 / 15}, & n: \text { odd }\end{cases}
$$

Then

$$
q_{n}=c_{n-1}^{2}= \begin{cases}14.1 / 15, & n: \text { even } \\ 1 / 15, & n: \text { odd }\end{cases}
$$

Hence $q_{n} q_{n+1}=14.1 /(15)^{2}>1 / 16$. By Corollary 2.2 , every solution of (2.9) is oscillatory.

Oscillation criteria in [1] are not valid for (2.9).

Define two sequences $\left\{R_{n}\right\}$ and $\left\{r_{n}\right\}$ as follows:

$$
\begin{aligned}
R_{n}= & q_{n}+q_{n-1}+q_{n} q_{n+1}+q_{n-1} q_{n-2}+q_{n} q_{n+1}^{2} \\
& +q_{n} q_{n+1} q_{n+2}+q_{n-1} q_{n-2}^{2}+q_{n} q_{n+1}^{2} q_{n+2} \\
& +q_{n-2}^{2} q_{n-1} q_{n-3}+q_{n-1} q_{n-2} q_{n-3}, \quad n \geq 4,
\end{aligned}
$$

and

$r_{n}=q_{n}+q_{n-1}+q_{n} q_{n+1}+q_{n-1} q_{n-2}, n \geq 3$.

THEOREM 2.2 Assume that there exists an increasing sequence $\left\{n_{k}\right\}$ such that $R_{n_{k}} \geq 1$. Then (1.1) is oscillatory.

Proof Suppose to the contrary, let (1.1) be nonoscillatory. Then (2.2) has a positive solution $\left\{s_{n}\right\}$ defined for $n \geq N$. From (2.2), by the iterating substitution, we have

$$
\begin{aligned}
1= & q_{n} q_{n+1} s_{n} s_{n+1}+q_{n}+q_{n+1}+1 /\left(s_{n-1} s_{n-2}\right) \\
= & q_{n} q_{n+1} s_{n} s_{n+1}\left(q_{n+2} s_{n+2}+1 / s_{n+1}\right) \\
& \times\left(q_{n+1} s_{n+1}+1 / s_{n}\right)+q_{n}+q_{n-1} \\
& +\left(q_{n-1} s_{n-1}+1 / s_{n-2}\right)\left(q_{n-2} s_{n-2}+1 / s_{n-3}\right) \\
& /\left(s_{n-1} s_{n-2}\right) \\
= & q_{n}+q_{n-1}+q_{n} q_{n+1}+q_{n-1} q_{n-2} \\
& +q_{n} q_{n+1}^{2} s_{n} s_{n+1}+q_{n} q_{n+1} q_{n+2} s_{n+1} s_{n+2} \\
& +q_{n} q_{n+1}^{2} q_{n+2} s_{n} s_{n+1}^{2} s_{n+2}+q_{n-2} /\left(s_{n-1} s_{n-2}\right) \\
& +q_{n-1} /\left(s_{n-2} s_{n-3}\right)+1 /\left(s_{n-1} s_{n-2}^{2} s_{n-3}\right) \\
> & q_{n}+q_{n-1}+q_{n} q_{n+1}+q_{n-1} q_{n-2}+q_{n} q_{n+1}^{2} \\
& +q_{n} q_{n+1} q_{n+2}+q_{n} q_{n+1}^{2} q_{n+2}+q_{n-1} q_{n-2}^{2} \\
& +q_{n-1} q_{n-2} q_{n-3}+q_{n-1} q_{n-2}^{2} q_{n-3} \\
= & R_{n}
\end{aligned}
$$

which contradicts the assumption. The proof is complete.

COROLlaRY 2.3 If $\lim \sup _{n \rightarrow \infty} R_{n}>1$, then (1.1) is oscillatory.

It is easy to see that $\lim \sup _{n \rightarrow \infty} r_{n}>1$, then $\lim \sup _{n \rightarrow \infty} R_{n}>1$.

Example 2.1 satisfies conditions of Corollary 2.3.

Remark 2.3 Corollary 2.3 improves Corollary 6.5.11 in [1].

\section{FORCED OSCILLATION}

We consider the forced equation

$$
\Delta^{2} x_{n}+p_{n} x_{n+1}=f_{n}, \quad n=0,1, \ldots,
$$

and the homogeneous equation

$$
\Delta^{2} x_{n}+p_{n} x_{n+1}=0 .
$$

Lemma 3.1 Let $\left\{\phi_{n}\right\}$ be a solution of (3.2) and $\left\{x_{n}\right\}$ be a solution of (3.1). Let $x_{n}=\phi_{n} y_{n}$, then $\left\{y_{n}\right\}$ satisfies

$$
\Delta\left(\phi_{n} \phi_{n+1} \Delta y_{n}\right)=\phi_{n+1} f_{n} .
$$


Proof Clearly,

$$
\phi_{n} \Delta x_{n}=\phi_{n} \Delta \phi_{n} y_{n}+\phi_{n} \phi_{n+1} \Delta y_{n} \text {. }
$$

Hence

$$
\begin{aligned}
& \Delta\left(\phi_{n} \phi_{n+1} \Delta y_{n}\right) \\
&= \Delta\left(\phi_{n} \Delta x_{n}\right)-\Delta\left(\phi_{n} \Delta \phi_{n} y_{n}\right) \\
&= \phi_{n+1} \Delta^{2} x_{n}+\Delta \phi_{n} \Delta x_{n}-\phi_{n+1} \Delta \phi_{n+1} \Delta y_{n} \\
&-\Delta\left(\phi_{n} \Delta \phi_{n}\right) y_{n} \\
&= \phi_{n+1}\left(f_{n}-p_{n} \phi_{n+1} y_{n+1}\right) \\
&+\Delta \phi_{n}\left(\Delta \phi_{n} y_{n}+\phi_{n+1} \Delta y_{n}\right) \\
&-\phi_{n+1} \Delta \phi_{n+1} \Delta y_{n}-\left(\phi_{n+1} \Delta^{2} \phi_{n}+\left(\Delta \phi_{n}\right)^{2}\right) y_{n} \\
&= \phi_{n+1} f_{n}-\phi_{n+1}^{2} y_{n+1} p_{n} \\
&+\phi_{n+1} \Delta y_{n}\left(\Delta \phi_{n}-\Delta \phi_{n+1}\right)-\phi_{n+1} \Delta^{2} \phi_{n} y_{n} \\
&= \phi_{n+1} f_{n}-\phi_{n+1}^{2} y_{n+1} p_{n} \\
&-\Delta^{2} \phi_{n} \phi_{n+1}\left(y_{n+1}-y_{n}\right)-\phi_{n+1} \Delta^{2} \phi_{n} y_{n} \\
&= \phi_{n+1} f_{n}-y_{n+1} \phi_{n+1}\left(p_{n} \phi_{n+1}+\Delta^{2} \phi_{n}\right) \\
&= \phi_{n+1} f_{n} .
\end{aligned}
$$

The proof is complete.

THEOREM 3.1 Let $\left\{\phi_{n}\right\}$ be a positive solution of (3.2). Assume that there exists a positive integer $N$ such that

(i)

$$
\liminf _{n \rightarrow \infty} \sum_{i=N}^{n} \phi_{i+1} f_{i}=-\infty
$$

and

$$
\limsup _{n \rightarrow \infty} \sum_{i=N}^{n} \phi_{i+1} f_{i}=\infty
$$

(ii)

$$
\sum_{i=N}^{\infty} \frac{1}{\phi_{i} \phi_{i+1}}=\infty
$$

(iii)

$$
\begin{aligned}
& \liminf _{n \rightarrow \infty} \sum_{i=N}^{n} \frac{1}{\phi_{i} \phi_{i+1}} \sum_{j=N}^{i-1} \phi_{j+1} f_{j}=-\infty, \\
& \limsup _{n \rightarrow \infty} \sum_{i=N}^{n} \frac{1}{\phi_{i} \phi_{i+1}} \sum_{j=N}^{i-1} \phi_{j+1} f_{j}=\infty .
\end{aligned}
$$

Then every solution of (3.1) is oscillatory.
Proof Suppose to the contrary, let $\left\{x_{n}\right\}$ be a positive solution of (3.1) and $x_{n}=\phi_{n} y_{n}$. By Lemma 3.1, $y_{n}$ satisfies (3.3).

Summing (3.3) from $N$ to $n-1$, we obtain

$$
\phi_{n} \phi_{n+1} \Delta y_{n}-\phi_{N} \phi_{N+1} \Delta y_{N}=\sum_{i=N}^{n-1} \phi_{i+1} f_{i} .
$$

Condition (i) implies that

$$
\liminf _{n \rightarrow \infty} \phi_{n} \phi_{n+1} \Delta y_{n}=-\infty \text {. }
$$

Let $N_{1}$ be a large integer that $\phi_{N_{1}} \phi_{N_{1}+1} \Delta y_{N_{1}}<$ $-M, M>0$. From (3.4), we obtain

$$
\begin{aligned}
\Delta y_{n} & =\frac{\phi_{N_{1}} \phi_{N_{1}+1} \Delta y_{N_{1}}}{\phi_{n} \phi_{n+1}}+\frac{1}{\phi_{n} \phi_{n+1}} \sum_{i=N_{1}}^{n-1} \phi_{i+1} f_{i} \\
& <-\frac{M}{\phi_{n} \phi_{n+1}}+\frac{1}{\phi_{n} \phi_{n+1}} \sum_{i=N_{1}}^{n-1} \phi_{i+1} f_{i} .
\end{aligned}
$$

Summing (3.5) from $N_{1}$ to $n-1$, we obtain

$$
\begin{aligned}
y_{n}-y_{N_{1}} \leq & -M \sum_{i=N_{1}}^{n-1} \frac{1}{\phi_{i} \phi_{i+1}} \\
& +\sum_{i=N_{1}}^{n-1} \frac{1}{\phi_{i} \phi_{i+1}} \sum_{j=N_{1}}^{i-1} \phi_{j+1} f_{j} .
\end{aligned}
$$

Condition (iii) and (3.6) imply that there exists a sequence $\left\{n_{i}\right\}$ such that $y_{n_{i}}<0$ for all large $i$, which is a contradiction.

We can prove this theorem in a similar manner for negative solutions of (3.1).

From (3.6), we obtain the following result.

THEOREM 3.2 Let $\left\{\phi_{n}\right\}$ be a positive solution of (3.2) with $\sum_{i=N}^{\infty} 1 /\left(\phi_{i} \phi_{i+1}\right)<\infty$. Assume that (iii) of Theorem 3.1 holds. Then every solution of (3.1) is oscillatory.

Example 3.1 Consider

$$
\begin{aligned}
\Delta^{2} x_{n} & +\frac{2}{(n+1)^{2}(n+3)} x_{n+1} \\
= & (-1)^{n} \frac{(2 n-1)(n+2)}{n+1}, \quad n=1,2, \ldots
\end{aligned}
$$


It is easy to see that the equation

$$
\Delta^{2} x_{n}+\frac{2}{(n+1)^{2}(n+3)} x_{n+1}=0
$$

has a solution $\left\{\phi_{n}=n /(n+1)\right\}$, which satisfies (ii). On the other hand,

$$
\sum_{i=N}^{n} \phi_{i+1} f_{i}=\sum_{i=N}^{n}(-1)^{i}(2 i-1)=(-1)^{n} n+c
$$

where $c$ is a constant. Then (3.9) implies that (i) is satisfied. Also, (iii) is satisfied. By Theorem 3.1, every solution of (3.7) is oscillatory.

Remark 3.1 Theorems 3.1 and 3.2 treat the oscillation of (3.1), which is caused by the forced term.

\section{References}

[1] R.P. Agarwal, Difference Equations and Inequalities, Marcel Dekker, NY, 1992.

[2] S.S. Cheng, Sturmian comparison theorems for three term recurrence equations, J. Math. Anal. Appl., 111 (1985), $465-474$.

[3] J.W. Hooker and W.T. Patula, Riccati type transformations for second order linear difference equations, J. Math. Anal. Appl., 82 (1981), 451-462.

[4] W.T. Patula, Growth and oscillation properties of second order linear difference equations, SIAM J. Math. Anal., 10 (1979), 1272-1279.

[5] L.H. Erbe and B.G. Zhang, Oscillation of second order linear difference equations, Chinese Math. J., 16(4) (1988), $239-252$.

[6] L.H. Erbe and B.G. Zhang, Oscillation of discrete analogues of delay equations, Diff. and Integral Equations, 2 (1989), 300-309. 


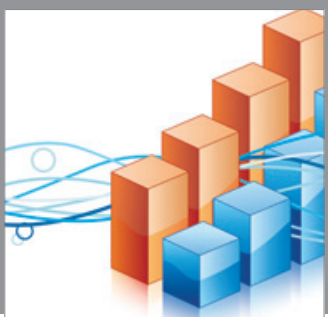

Advances in

Operations Research

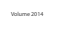

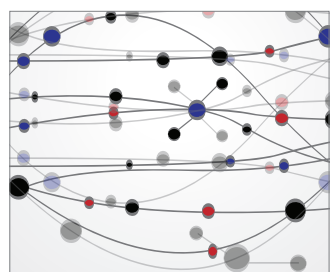

\section{The Scientific} World Journal
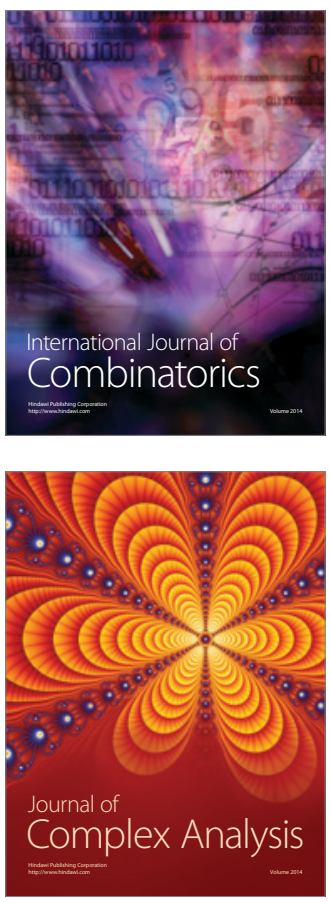

International Journal of

Mathematics and

Mathematical

Sciences
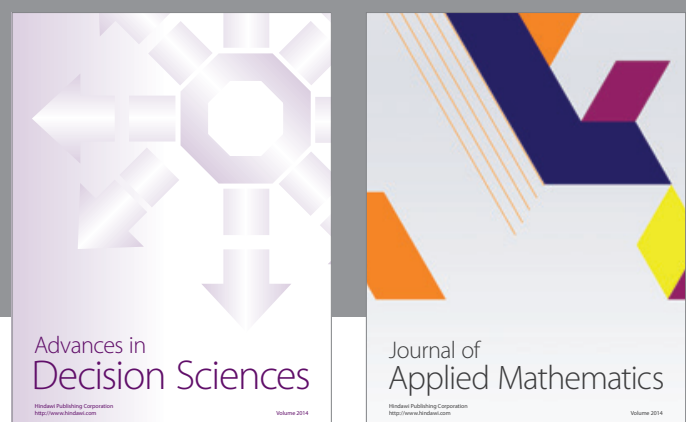

Journal of

Applied Mathematics
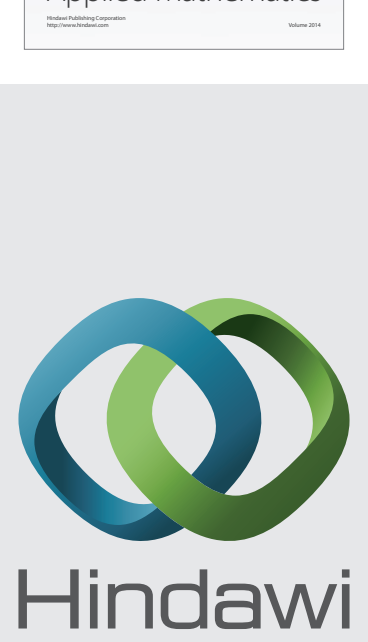

Submit your manuscripts at http://www.hindawi.com
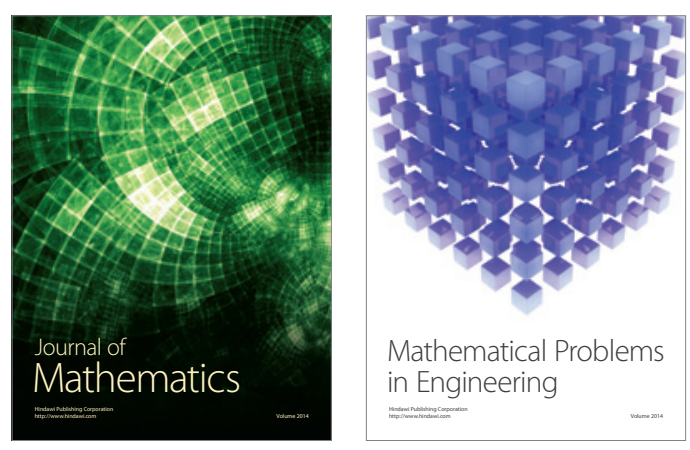

Mathematical Problems in Engineering
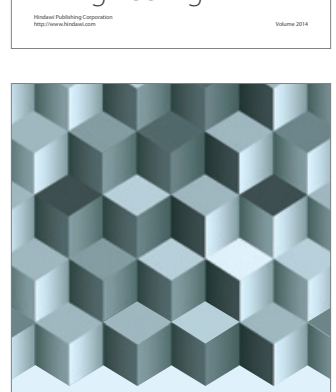

Journal of

Function Spaces
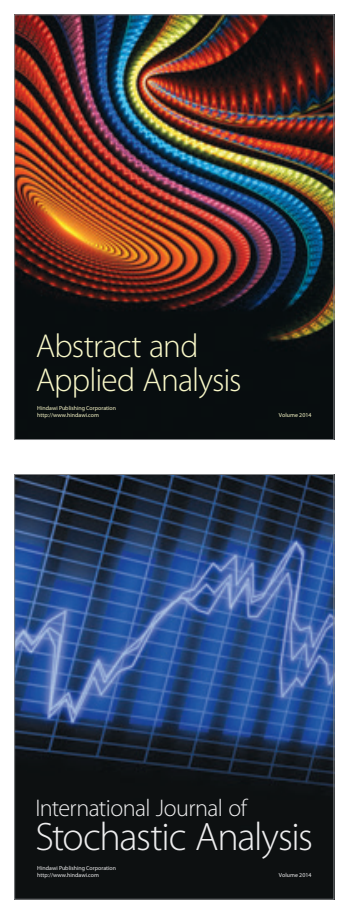

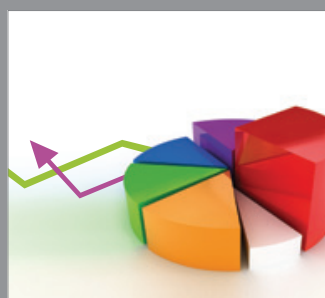

ournal of

Probability and Statistics

Promensencen
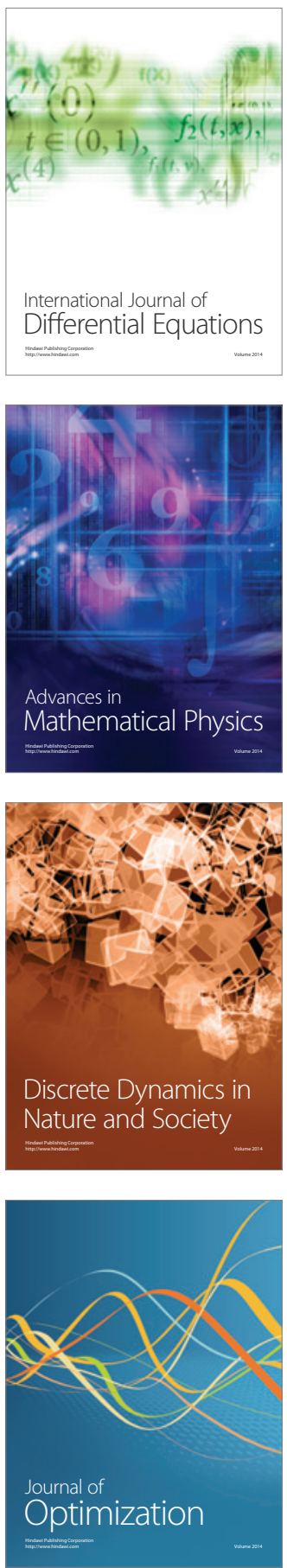